\title{
Author Correction: Cryo-EM structure of the exocyst complex
}

Kunrong Mei, Yan Li, Shaoxiao Wang, Guangcan Shao, Jia Wang, Yuehe Ding, Guangzuo Luo, Peng Yue, Jun-Jie Liu, Xinquan Wang, Meng-Qiu Dong (iD, Hong-Wei Wang and Wei Guo iD

Correction to: Nature Structural \& Molecular Biology https://doi.org/10.1038/s41594-017-0016-2, published online 15 January 2018.

In the version of this article originally published, the value given for electron dose in Table 1 was incorrect. This value was originally stated as 4.8 but should have been 50 . The error has been corrected in the HTML and PDF versions of the article.

Published online: 5 November 2018

https://doi.org/10.1038/s41594-018-0157-y 